\title{
Hyperpolarizability studies of some nonconjugated twin donor-acceptor molecules
}

\author{
ELIZABETH CHIRACKAL VARKEY and KRISHNAPILLAI SREEKUMAR* \\ Department of Applied Chemistry, Cochin University of Science and Technology, Cochin 22, India
}

MS received 12 April 2010

\begin{abstract}
Extensive theoretical calculation on the effects of spacer length enhancement on the second-order NLO properties of twin donor acceptor molecules having two amide units bridged by the $\mathrm{CH}_{2}$ spacers was performed. The role of such aliphatic bridges on the Donor-Acceptor groups was computed by ZINDO/CV quantum chemical formalism. The odd-even effects were observed in twin donor acceptor systems (with two aliphatic units) linked by an alkyl spacer of varying length from $n=1$ to $n=12$. The system considered for the present study was $N, N^{\prime}$-alkane-(1, n) diyl bis-4-hydroxy hexanamides. For an odd number of $\mathrm{CH}_{2}$ spacers, the $\beta$ value was an order of magnitude higher than that for the even number of $\mathrm{CH}_{2}$ spacers. The origin for such oscillation is attributed to the similar oscillations in the dipole moment difference between the ground state and the dipole allowed state and to some extent on the variation in the oscillator strength.
\end{abstract}

Keywords. Nonlinear optics; oscillator strength; optical gap; polarizability.

\section{Introduction}

The design of materials with large nonlinear optical (NLO) responses has been a topic of active research because of their potential applications in a number of advanced optoelectronic devices (Pal et al 2008). The development of organic second-order NLO materials is motivated by the promising performance and cost improvements related to telecommunications, computing, embedded network sensing, signal transmission, and many other optical applications (Park et al 2004). It is well-known that second-order NLO properties originate from the noncentrosymmetric alignment of NLO chromophores (generally achieved under an electric field), either doped as a guest or covalently introduced to the polymer systems, although the strong intermolecular dipole-dipole interactions among the chromophore moieties in the polymeric system make the poling-induced noncentrosymmetric alignment of chromophores a daunting task (Li et al 2009).

Nonlinear optics deals with the interaction of electromagnetic fields in various media to produce new fields altered in phase, frequency, amplitude or other propagation characteristics from the incident fields. When a beam of light is impinged on a material it causes the charges of the atom to oscillate. In a linear material the amount of charge displacement is proportional to the instantaneous magnitude of the electric field. The charges oscillate at

\footnotetext{
*Author for correspondence (ksk@cusat.ac.in)
}

the same frequency as the incident light. The oscillating charges either radiate light at that frequency or the energy is transferred into non-radiative modes that result in material heating or other energy transfer mechanisms. With small fields the displacement of charge from the equilibrium position, polarization $(P)$, will be proportional to the applied field, $E$.

$$
P=\alpha E,
$$

where $\alpha$-is the linear polarizability.

In a nonlinear optical material, the displacement of charge from its equilibrium value is a nonlinear function of the electric field. When exposed to laser light, i.e. very high intensity electric field, the polarizability of all materials can be drawn beyond a linear regime (Gunter 2000; Robert and Boyd 2009).

Nonlinear polarization

$$
P=P_{0}+\chi^{(1)} E+\chi^{(2)} E^{2}+\chi^{(3)} E^{3}+\cdots .
$$

In this paper, a theoretical analysis of the nonlinear optical properties of the twin donor acceptor systems is presented using the established ZINDO (SOS)/CV quantum chemical formalism. Even though, ab initio-CPHF computations are numerically more accurate and precise, the results are not easily amenable to chemical interpretation. Since they are based on derivative rather than SOS formalism, the interpretation of computed response is difficult, albeit of their accuracy in predicting trends. Also, in experimental measurements, electrons will go to the higher excited states and the higher level contribution may not be too small to neglect. Almost all the semiem- 
pirical SOS procedures provide second order responses in reasonable agreement with experiments. Moreover, they permit a basic understanding of the origin of the NLO response in a chemical sense, by identifying the molecular excited states primarily responsible for an NLO response within the SOS formalism (Kanis et al 1994).

\section{Twin $D-A$ system for NLO study}

The molecular systems considered for the NLO study were the amido diols which were experimentally synthesized by the aminolysis of $\varepsilon$-caprolactone by using the diamino alkanes (Guass 2000). The electron acceptor groups and donor groups were attached to a nonconjugated system and the donor-acceptor systems show charge transfer between electron donating and electron withdrawing groups and hence develop the polarization in the system. By designing the effective NLO chromophores (highly active chromophore molecules which have a large molecular hyperpolarizability $\beta$ ), which can be incorporated into a polymer chain either in the main chain or side chain by covalent linkages one could develop polymer systems with high second harmonic generation efficiency. The twin donor-acceptor system $(D-A-A-D)$ and single donor-acceptor system $(D-A)$ selected for the NLO study are shown in the figure 1.

In this article we have tried to examine the dipole moment, polarizability, hyperpolarizability, oscillator strength and optical gaps of the twin donor acceptor systems by varying the number of alkyl groups between $D-A$ and $A-D$ chromophores.

\section{Methodology}

Figure 1 shows the molecular systems considered for the present study. All the geometries have been optimized using the AM1 parametrized Hamiltonian available in the Gaussian 03 set of codes (Guass 2000; Yongjun et al 2000; Gaussian 2003). Some of the compounds (with smaller alkyl units) have already been synthesized in good yields and well characterized (Elizabeth and Sreekumar 2008). The geometries obtained by the AM1 calculations have been compared with geometries obtained using the

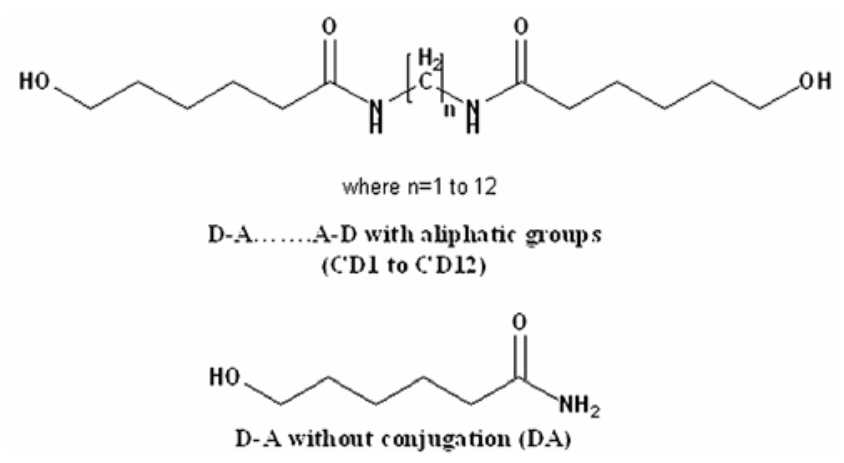

Figure 1. Twin donor acceptor systems.
HF based methods for the smaller chromophore with $n=2$. The geometries obtained by the HF and AM1 methods have similar bond lengths and bond angles. Therefore, it is believed that the AM1 Hamiltonian is quite reliable for these systems and thus have proceeded with it for bigger systems with size up to $n=12$. These optimized geometries were used to compute the NLO properties using the Zerner's INDO method (Datta and Pati 2005a; Davis et al 2005). The level of CI calculations, were taken with singles (SCI) to obtain a reliable estimate of the second order optical response. The reference determinants chosen are dominant in the description of the ground state and the lowest one-photon excited states. For each reference determinant, 8 occupied and 8 unoccupied molecular orbitals were used to construct a CI space with configuration dimension of 900. To calculate NLO properties, the correction vector method was used, which implicitly assumes all the excitations to be approximated by a correction vector (Datta and Pati 2005b; Dewar et al 1985). The CI approach adopted here has been extensively used in earlier works and provided excitation energies and dipole matrix elements in good agreement with experiment. The polarizabilities were also computed using the sum-over-states (SOS) formalism, and values similar to those provided by the $\mathrm{CV}$ method were obtained. All the calculations have been performed at a frequency of $1064 \mathrm{~nm}(1.17 \mathrm{eV})$ corresponding to the Nd:YAG laser. Details of this method have been published in a number of papers (Albert and Ramasesha 1990; Ramasesha and Albert 1990; Ramasesha et al 1995; Datta and Pati 2004, 2005a, 2006).

\section{Results and discussion}

The change in dipole moment $(\Delta \mu)$ (the difference in dipole moments between the ground state $\left(\mu_{\mathrm{g}}\right)$ and the lowest energy dipole allowed state $\left(\mu_{\mathrm{t}}\right)$ ), oscillator strength $(\phi)$, polarizability $(\alpha)$, hyperpolarizability $(\beta)$, percentage of chirality $\left(\beta_{x y z}\right)$, and the optical $\operatorname{gap}(\delta E)$ (the energy difference between the ground state and the lowest energy dipole allowed state) were calculated for the molecules of spacer length from $n=1$ to $n=12$. The corresponding values obtained by the dynamic SOS and correction vector method are shown in the tables 1 and 2 .

For the twin donor-acceptor system, the even chains have very little dipole moment $(\approx 3 D)$ while the odd ones have much higher dipole moment $(\approx 12 D)$. The dipole moment for the single $D-A$ molecule, is calculated as, $\mu_{\mathrm{g}}$ $\approx 7 \cdot 3 \mathrm{D}$. For a perfect parallel arrangement of the dipoles, the classical non-interacting picture predicts the total dipole moment as twice the single $D-A$ molecule value for parallel arrangement and 0 for a perfect anti-parallel arrangement. While, for the even spacers the dipole moments are nearer to zero, the odd-spacers show much smaller values from the classical result of twice the single 
Table 1. The ground state dipole moment, transition dipole moment, the change in dipole moment, polarizability, hyperpolarizability, percentage of chirality and $\mathrm{EFISH} \beta$.

\begin{tabular}{|c|c|c|c|c|c|c|c|}
\hline System & $\begin{array}{l}\mu_{\mathrm{g}} \\
\text { (D) }\end{array}$ & $\begin{array}{l}\mu_{\mathrm{t}} \\
(\mathrm{D})\end{array}$ & $\begin{array}{l}\Delta \mu \\
(\mathrm{D})\end{array}$ & $\left(10^{-24} \mathrm{esu}\right)$ & $\begin{array}{c}\beta \\
\left(10^{-32} \mathrm{esu}\right)\end{array}$ & $\begin{array}{c}\beta_{\mathrm{xyz}} \\
\left(10^{-32} \mathrm{esu}\right)\end{array}$ & $\begin{array}{c}\mu \beta \\
\left(10^{-32} \mathrm{esu}\right)\end{array}$ \\
\hline CD1 & $12 \cdot 0158$ & $3 \cdot 1567$ & $8 \cdot 8591$ & $18 \cdot 6263$ & $76 \cdot 7197$ & $0 \cdot 1153$ & $903 \cdot 2722$ \\
\hline CD2 & $3 \cdot 4912$ & $4 \cdot 5454$ & 1.0542 & $18 \cdot 1112$ & $3 \cdot 2928$ & $0 \cdot 1587$ & $6 \cdot 2674$ \\
\hline CD3 & $12 \cdot 3109$ & $3 \cdot 3943$ & $8 \cdot 9166$ & 17.7776 & $89 \cdot 1017$ & $0 \cdot 1587$ & $1065 \cdot 3001$ \\
\hline CD4 & 3.4869 & $4 \cdot 5681$ & 1.0812 & $17 \cdot 8422$ & $2 \cdot 5068$ & $0 \cdot 1614$ & 4.6629 \\
\hline CD5 & $12 \cdot 3344$ & 3.4397 & $8 \cdot 8947$ & 17.7882 & $94 \cdot 1268$ & $0 \cdot 1743$ & 1134.6722 \\
\hline CD6 & 3.4786 & 4.5910 & $1 \cdot 1124$ & $18 \cdot 2114$ & $2 \cdot 3422$ & $0 \cdot 1504$ & $6 \cdot 8692$ \\
\hline CD7 & $12 \cdot 3432$ & $3 \cdot 4481$ & $8 \cdot 8951$ & 18.4991 & 95.8396 & $0 \cdot 1370$ & $1136 \cdot 1731$ \\
\hline CD8 & $3 \cdot 5174$ & $4 \cdot 0483$ & 0.5309 & $18 \cdot 5704$ & $14 \cdot 3541$ & 0.2035 & $28 \cdot 1434$ \\
\hline CD9 & $12 \cdot 3144$ & 3.4141 & 8.9003 & $18 \cdot 6107$ & $98 \cdot 1683$ & $0 \cdot 2441$ & $1142 \cdot 6082$ \\
\hline CD10 & $3 \cdot 5232$ & $3 \cdot 3031$ & $0 \cdot 2201$ & $18 \cdot 5829$ & $16 \cdot 1086$ & $0 \cdot 2427$ & $30 \cdot 5099$ \\
\hline CD11 & $12 \cdot 3075$ & $3 \cdot 3809$ & 8.9266 & $18 \cdot 6232$ & $99 \cdot 0828$ & $0 \cdot 2560$ & $1149 \cdot 1025$ \\
\hline CD12 & $3 \cdot 5231$ & $3 \cdot 3072$ & $0 \cdot 2159$ & $18 \cdot 6409$ & $17 \cdot 7050$ & 0.2221 & 33.0583 \\
\hline DA & $7 \cdot 30133$ & $3 \cdot 2115$ & 4.0898 & $16 \cdot 0207$ & $16 \cdot 5492$ & 0.4866 & $33 \cdot 5304$ \\
\hline
\end{tabular}

Table 2. Oscillator strength $(f)$, the optical gap $(\delta E)$ and the distance between the dipoles $(d)$.

\begin{tabular}{lccc}
\hline System & $f$ & $\begin{array}{c}\delta E \\
(\mathrm{eV})\end{array}$ & $\begin{array}{c}d \\
(\AA)\end{array}$ \\
\hline CD1 & $0 \cdot 2547$ & $6 \cdot 729$ & $2 \cdot 3608$ \\
CD2 & $0 \cdot 5194$ & $6 \cdot 6185$ & $3 \cdot 7316$ \\
CD3 & $0 \cdot 2911$ & $6 \cdot 6532$ & $4 \cdot 9649$ \\
CD4 & $0 \cdot 5249$ & $6 \cdot 6220$ & $6 \cdot 2635$ \\
CD5 & $0 \cdot 2979$ & $6 \cdot 6281$ & $7 \cdot 5336$ \\
CD6 & $0 \cdot 5301$ & $6 \cdot 6215$ & $8 \cdot 8141$ \\
CD7 & $0 \cdot 2988$ & $6 \cdot 6175$ & $10 \cdot 1048$ \\
CD8 & $0 \cdot 4111$ & $6 \cdot 6050$ & $11 \cdot 3934$ \\
CD9 & $0 \cdot 2928$ & $6 \cdot 6144$ & $12 \cdot 6663$ \\
CD10 & $0 \cdot 2739$ & $6 \cdot 6096$ & $13 \cdot 9620$ \\
CD11 & $0 \cdot 2871$ & $6 \cdot 6123$ & $15 \cdot 2297$ \\
CD12 & $0 \cdot 2746$ & $6 \cdot 6104$ & $16 \cdot 5282$ \\
DA & $0 \cdot 2749$ & $7 \cdot 0173$ & $6 \cdot 4843$ \\
\hline
\end{tabular}

$D-A$ system value. Such a trend can be easily understood: for the even chains, the dipoles are staggered and almost perfectly anti-parallel, however, for the odd chains, even though the orientations are eclipsed, the dipoles are not exactly parallel because of the $s p^{3}$ hybridization along the alkyl principle axis (Datta and Pati 2005b). The even chains have very little change in dipole moment $(\Delta \mu \approx 1 D)$ while the odd ones have much higher change in dipole moment $(\Delta \mu \approx 8 D)$. The change in dipole moment for the single $D-A$ molecule, is calculated as, $\Delta \mu \approx 4 D$. Hence for the twin donor-acceptor system a strong odd even effect was observed in the case of dipole moments and change in dipole moments.

To understand the linear and nonlinear optical properties, the polarizability $\alpha$ and first frequency dependent hyperpolarizability $\beta$ was calculated with varying chain lengths for the $D-A-A-D$ systems. The electronic polarizability is defined as the ratio of the induced dipole moment of a system to the applied electric field that produces the dipole moment. For systems where the electric field induced polarization is not constrained only to the direction of applied field, polarizability is a tensor of rank 2. Kleinman's symmetry relations allows us to simplify the calculations of the tumbling average of the polarizability from the tensors as follows (Pal et al 2008).

$$
\alpha=\frac{1}{3}\left(\alpha_{\mathrm{XX}}+\alpha_{\mathrm{YY}}+\alpha_{\mathrm{ZZ}}\right)
$$

In the case of the twin donor-acceptor system the polarizability $(\alpha)$ value was constant in all the odd-even series $\left(\approx 18 \times 10^{-24}\right)$, while the single donor acceptor system possesses a polarizability value slightly lesser than the twin donor-acceptor system. No odd-even effects were observed on the polarizability parameters of the twin donor-acceptor systems.

The microscopic first hyperpolarizability, $\beta$ is a $3 \mathrm{rd}$ rank tensor, which has 27 tensor components. But in the general case of a molecule with no symmetry, these 27 elements are reduced to 10 according to Kleinman symmetry for second harmonic generation $\beta(2 \omega ; \omega, \omega)$. Due to independent interchange of Cartesian coordinates, $\beta_{i j k}=\beta_{i k j}$. These irreducible 10 tensor components are $\beta_{x x x}, \beta_{x x y}, \beta_{x y y}, \beta_{y y y}, \beta_{x x z}, \beta_{x y z}, \beta_{y y z}, \beta_{x z z}, \beta_{y z z}$ and $\beta_{z z z}$. By taking the tensor product of these 10 tensor components, orientationally averaged hyperpolarizabilities can be calculated from the equations given below (Ouder et al 1977; Ouder and Chemla 1982; Bishop 1998; Pati et al 1999, 2001; Pal et al 2008).

$$
\beta_{\mathrm{vec}}(-2 \omega ; \omega, \omega)=\sqrt{\beta_{\mathrm{X}}^{2}+\beta_{\mathrm{Y}}^{2}+\beta_{\mathrm{Z}}^{2}}
$$

where

$$
\beta_{i}=\sum_{j=x, y, z} \frac{\beta_{i j j}+\beta_{j i j}+\beta_{j j i}}{3} ; i=x, y, z .
$$

In an electric dipole approximation, the tensor component $\beta_{x y z}$, is the chiral component of the medium and all other 
nine tensor components are achiral. Because of the property of symmetric tensor product basis set elements, the value of $\beta_{x y z}$ becomes zero in the tensor product. But the chiral component $\beta_{x y z}$ becomes non-zero and contributes a major portion to the macroscopic $(3 D)$ second order polarizability tensor Thus, while designing the molecule with high $\beta$ value, the $\beta_{x y z}$ component becomes important in macroscopic perception. The molecules which are more symmetric (less chiral) will have small values of the chiral component $\beta_{x y z}$ (Ouder and Zyss 1982)

The hyperpolarizability factor shows similar trends as the dipole moment for $D-A-\left(\mathrm{CH}_{2}\right)_{n}-A-D$ system from $n=1$ to $n=12$. Odd numbered $-\mathrm{CH}_{2}-$ systems had high hyperpolarizability value $\left(76-100 \times 10^{-32} \mathrm{esu}\right)$ and even numbered $-\mathrm{CH}_{2}-$ systems possess low hyperpolarizability $\left(2-17 \times 10^{-32} \mathrm{esu}\right)$. The $D-A$ system with aliphatic groups is also having $D-(\mathrm{CH})_{5}-A$, an odd number spacer, it possesses a low value of $\beta\left(16.5 \times 10^{-32} \mathrm{esu}\right)$ compared to the other odd numbered species. But it possesses a higher value than the even numbered species. The EFISH $\beta$ values also show strong odd-even oscillation phenomena.

Ouder and Chemla (1977) established a link between $\beta$ and the details of a low-lying charge-transfer transition through the two-level model. NLO properties of organic charge transfer complexes like $D-\pi-A$ can be captured very nicely by this model (Bishop 1998; Pati et al 2001). This model assumes that the electronic properties of the molecules are determined by the ground state and a low energy charge transfer excited state. Polarization results primarily from the mixing of charge transfer state with the ground state through the interaction of the molecule with the electric field. The sum over states (SOS) formalism lies within the limit of two level model, when the contribution from higher excited states are negligible compared to the first excited state (Kanis et al 1994). From the two-level model, using electric dipole approximation, the second harmonic generation response ( $\mathrm{SHG}$ ) can be written as (Pati et al 2001)

$$
\beta_{\text {two-level }}=\frac{3 e^{2}}{2 \hbar} \frac{\omega_{12} f \Delta \mu}{\left(\omega_{12}^{2}-\omega^{2}\right)\left(\omega_{12}^{2}-4 \omega^{2}\right)},
$$

where $\omega_{12}$ is the excitation energy, $f$ is the oscillator strength, $\Delta \mu$ is the difference between the dipole moments of the ground and the excited state, and $\omega$ specifies the excitation frequency of the oscillating electric field. The most important factor in the above simple expression is that the SHG coefficient is directly proportional to the oscillator strength and the dipole moment difference and is inversely proportional to the optical gap. Thus, any phenomenon that decreases the gap or increases the dipole moment difference between the ground and the excited state or increases the oscillator strength will enhance $\beta$. But as seen from table 2, the optical gap $(\delta E)$ remains almost constant along the series and shows no such odd-even oscillations. In fact, the oscillator strength for the even chromophores is slightly larger than that for the odd ones. Thus, the only factor that governs such an odd-even oscillation is $\Delta \mu$. Figure 2 shows the variation of $\Delta \mu$ with increase in the spacer length. One can clearly see the odd-even variation in $\Delta \mu$ similar to that observed for $\beta$. This is to say that the excited state polarization has a strong dependence on the interchromophoric arrangements (Dewar et al 1985; Datta and Pati 2004, 2005a, b, 2006).

The oscillator strength is a dimensionless quantity to express the relative strength of the electronic transitions within atomic and molecular systems. The oscillator strength shows remarkable features in which $f$-increases for even numbered spacers and decreases for odd numbered spacers. A uniform increase is observed in even numbered spacers and decrease is observed in odd numbered spacers up to the spacer length of $n=8$. But after the spacer $n=8$ the odd-even oscillations were not that pronounced compared to the spacer less than eight. This indicates that the spacer above $n=8$ had little influence on the electronic transitions. Hence, it was found that the charge transfer is predominant only up to spacer $=7$. The optical gap is calculated as the energy difference between the geometry relaxed ground state and the lowest optically allowed state with substantial oscillator strength (Simpson 2004). The optical gap of the systems were very high in a range of 6-7 eV. A high optical gap was observed in the $D-A$ system which prevented any coupling between donor and acceptor groups and resulted in the reduction of polarization effects.

The distance between the dipoles increases as the number of alkyl units increases. The distance between the dipoles also exhibit an influence on the odd-even phenomena. The distance between the even dipoles is more than their odd counterparts as the even ones have a centrosymmetric arrangement which increases their interchromophoric distances (Datta and Pati 2005b, 2006). For each even spacer distance, $\beta$ is smaller and for each odd spacer distance $\beta$ is larger.

\section{Conclusions}

Semiempirical calculations were performed to understand the NLO efficiency of donor-acceptor systems with saturated spacers ( $n=1$ to $n=12)$ and the odd-even oscillation was observed. The NLO responses of the chromophores are mainly due to the electrostatic interaction on the strengths of the donor $(D)$ and acceptor $(A)$. For the saturated bridged molecules, electrons are localized at the respective sites and thus the electron transfer has a much shorter range. Hence the hyperpolarizability of saturated $D-A$ systems were low compared to the conjugated NLO chromophores. The single $D-A$ system behaves 

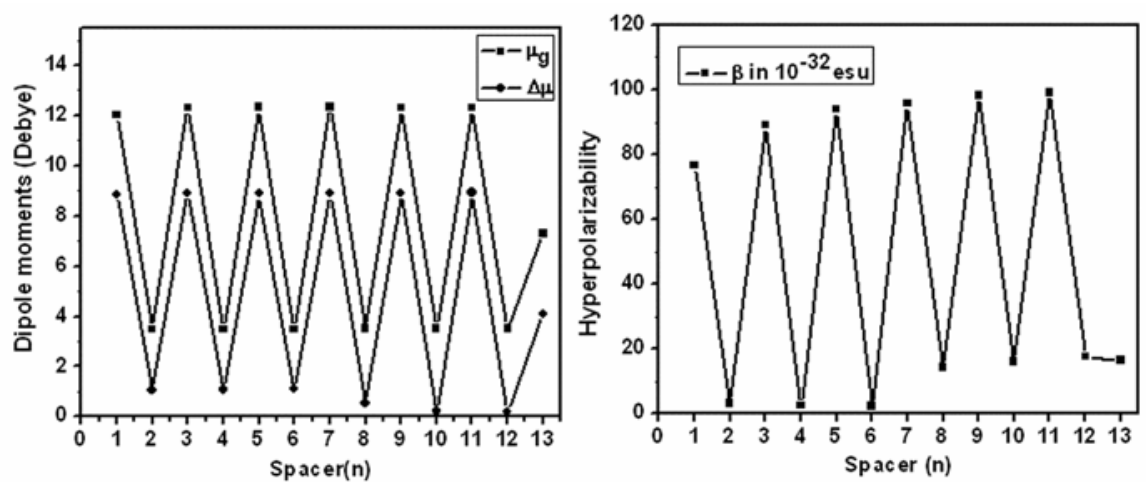

Figure 2. Pictorial representation of odd-even effects observed in ground state dipole moment $\left(\mu_{\mathrm{g}}\right.$ in debye), the change in dipole moment ( $\Delta \mu$ in debye) and hyperpolarizability $\left(\beta\right.$ in $10^{-32}$ esu).

independent of each other, and there was no possibility for intermixing between the chromophores. It was observed that while the linear polarizability remained almost constant throughout the series, the ground state dipole moment, change in dipole moment, EFISH $\beta$ and the first hyperpolarizability $(\beta)$ showed strong odd-even oscillations. The hyperpolarizability value $(\beta)$ showed an order of magnitude increase for odd spacers compared to the even spacers. The significantly higher SHG efficiencies for the twin $D-A$ systems of odd series implies that there is a less effective cancellation of dipoles associated with the chromophores of the odd dimers compared to the even ones. In the even cases, the all trans arrangement of the alkyl spacer of the twin molecule will lead to conformers that possess a centre of symmetry, while in the odd cases it could lead to structures which may possess a mirror plane. The odd and even spacer series separately show the values to be almost constant with the increase of odd $-\mathrm{CH}_{2}$ spacers and even $-\mathrm{CH}_{2}$ spacers. The origin for such oscillations is attributed to the similar oscillations in the ground state dipole moment, dipole moment difference between the ground state and the dipole allowed excited state and to some extent on the variation in the oscillator strength. This arises due to the change in the dipolar orientations between the staggered and eclipsed form for the even and odd chains, respectively.

\section{Acknowledgements}

The authors wish to thank Prof Swapan K Pati, Sudipta dutta (JNCASR, Bangalore) and Dr Ayan Datta of IISER, Trivandrum for useful discussions related to ZINDO calculations. The authors gratefully acknowledge financial support from DRDO, India for the research grants.

\section{References}

Albert I D L and Ramasesha S 1990 J. Phys. Chem. 946540 Bishop D M 1998 Adv. Chem. Phys. 1041

Datta A and Pati S K 2004 J. Phys. Chem. A108 320

Datta A, Pati S K, Davis D and Sreekumar K 2005 J. Phys. Chem. A109 4112

Datta A and Pati S K 2005a J. Molecular Structure, Theochem. 75697

Datta A and Pati S K 2005b Chem. Eur. J. 114961

Datta A and Pati S K 2006 Chem. Soc. Rev. 351305

Davis D, Sreekumar K and Pati S K 2005 Synth. Met. 155384

Dewar M J S, Zoebisch E G, Healy E F and Stewart J J P 1985 J. Am. Chem. Soc. 1073902

Elizabeth C V and Sreekumar K 2008 Proceedings SAMPADA2008, Pune, India

Gaussian 03 Revision B.05 Gaussian Inc. Pittsburgh P A 2003

Guass J 2000 Modern methods and algorithms of quantum chemistry, Proceedings, 2nd edn 3541

Gunter P 2000 Nonlinear Optical Effects Mater. 7211

Kanis D R, Ratner M A and Marks T J 1994 Chem. Rev. 94195

Li Z, Yu G, Wu W, Liu Y, Ye C, Qin J and Li Z 2009 Macromolecules 423864

Ouder J L and Chemla D S 1977 J. Chem. Phys. 662664

Ouder J L and Zyss J 1982 Phys. Rev. A26 2016

Pal S, Manna A K and Pati S K 2008 J. Chem. Phys. 129204301

Park G, Jung W S and Ra C S 2004 Bull. Korean Chem. Soc. 25 1427

Pati S K, Marks T J and Ratner M A $2001 \mathrm{~J}$. Am. Chem. Soc. 1237287

Pati S K, Ramasesha S, Shuai Z and Bredas J L 1999 Phys. Rev. B59 14827

Ramasesha S and Albert I D L 1990 Phys. Rev. B42 8587

Ramasesha S, Shuai Z and Bredas J L 1995 Chem. Phys. Lett. 245224

Robert W and Boyd J 2009 Biomed. Opt. 1429902

Simpson G J 2004 Chem. Phys. Chem. 51301

Yongjun L, Ying L, Xianghe K, Min X and Chengbu L 2000 Theoret. Exp. Chem. 36333 